University of Nebraska - Lincoln

DigitalCommons@University of Nebraska - Lincoln

6-14-2006

\title{
Solution chemistry control to make well defined submicron continuous fibres by electrospinning: the $(\mathrm{CH} 3 \mathrm{CH} 2 \mathrm{CH} 2 \mathrm{O}) 4 \mathrm{Ti} /$ $\mathrm{AcOH} /$ poly(N-vinylpyrrolidone) system
}

\author{
Gustavo F. Larsen \\ University of Nebraska-Lincoln, glarsen1@unl.edu \\ Maciej Skotak \\ University of Nebraska-Lincoln, m.skotak@gmail.com
}

Follow this and additional works at: https://digitalcommons.unl.edu/chemengmatsci

Part of the Materials Science and Engineering Commons

Larsen, Gustavo F. and Skotak, Maciej, "Solution chemistry control to make well defined submicron continuous fibres by electrospinning: the $(\mathrm{CH} 3 \mathrm{CH} 2 \mathrm{CH} 2 \mathrm{O}) 4 \mathrm{Ti} / \mathrm{AcOH} /$ poly $(\mathrm{N}$-vinylpyrrolidone) system" (2006). Papers in Materials Science. 5.

https://digitalcommons.unl.edu/chemengmatsci/5

This Article is brought to you for free and open access by the Chemical and Biomolecular Engineering Research and Publications at DigitalCommons@University of Nebraska - Lincoln. It has been accepted for inclusion in Papers in Materials Science by an authorized administrator of DigitalCommons@University of Nebraska - Lincoln. 


\title{
Solution chemistry control to make well defined submicron continuous fibres by electrospinning: the $\left(\mathrm{CH}_{3} \mathrm{CH}_{2} \mathrm{CH}_{2} \mathrm{O}\right)_{4} \mathrm{Ti} / \mathrm{AcOH} /$ poly $(N$-vinylpyrrolidone $)$ system
}

\begin{abstract}
The $\left(\mathrm{CH}_{3} \mathrm{CH}_{2} \mathrm{CH}_{2} \mathrm{O}\right)_{4} \mathrm{Ti} / \mathrm{AcOH} /$ poly$(N$-vinylpyrrolidone) system has been shown to yield suitable formulations for electrohydrodynamic (EHD) processing into continuous fibres and particles. The EHD processability of this, and generally most sol-gel-based formulations, into well defined fibres and particles with narrow submicron-range diameter distributions depends not only on the EHD process variables (electric field and flow rates), but also on the stability of key physical properties (e.g., conductivity, viscosity, surface tension, density and dielectric constant) of the sol over time. Sols that were almost certainly still undergoing hydrolysis and condensation reactions have been processed via EHD by many research groups to make materials with submicron features. This paper thus highlights the need for understanding the chemistry of EHDprocessed solutions to afford reproducibility and near monodispersity in fibre diameter. Reactions were monitored over time with the aid of a flow-through infrared cell, and the structure of species in solution is discussed. Conductivity and viscosity changes with time of representative formulations are discussed in the light of typical EHD processing time scales. Representative sols were processed via EHD, and woven and non-woven fibrous mats were characterized by scanning electron microscopy (SEM).
\end{abstract}

Keywords: Electrohydrodynamic (EHD), electrospinning.

Comment: This paper was originally published in the Journal of "Materials Chemistry" Vol 16. Page 3031-3039 on $14^{\text {th }}$ June 2006 .All the copyrights (C) of this paper belongs to "The Royal Society of Chemistry". 


\subsection{Introduction}

The use of electrohydrodynamic (EHD) concepts to make fibres (electrospinning),and particles (electrospray)from suitable precursors in solution is rooted in the pioneering work of Lord Rayleigh, ${ }^{1}$ Zelenky, ${ }^{2}$ and a series of U.S patents by Formhals that date back to 1930 's and 1940's. ${ }^{3}$ In the mid 1990's Reneker's group reinvigorated the electro spinning research field. A typical EHD experimental setup is depicted in Fig.1.A liquid containing adequate amounts of a dissolved fibre- or particle forming precursor is pumped through a capillary and a collector surface is applied .When the experiment is started the electric field generally creates a conical meniscus ("Referred to as the "Taylor cone") from the growing droplet pending from the capillary(Fig.1,inset).A thin ,electrified liquid jet is then ejected off the tip of the Taylor cone, as a natural means for the whole system to close the electrical circuit. Depending on the values adopted for the process variables and the physical properties of the solution (which in turn determine the ease by which the electrified liquid jet may undergo break-up into an electrospray), and the rate of solvent evaporation during the time-of -flight of the electrified liquid jet en route to the collector electrode, either fibres or the particles are obtained .In the interest of brevity ,structures that depart from this standard cone-jet scenario will not be introduced. ${ }^{6}$

Models that may ultimately allow prediction of the diameter of electro spun fibres are now available ${ }^{7}$.Application and future refinement of these models logically relies on knowledge of relevant physical variables of the EHD-processed solution ,such as electrical conductivity, viscosity , dielectric constant, density, and surface tension, as well as on accurate control of the processing variables such as applied electric field and flow rate.

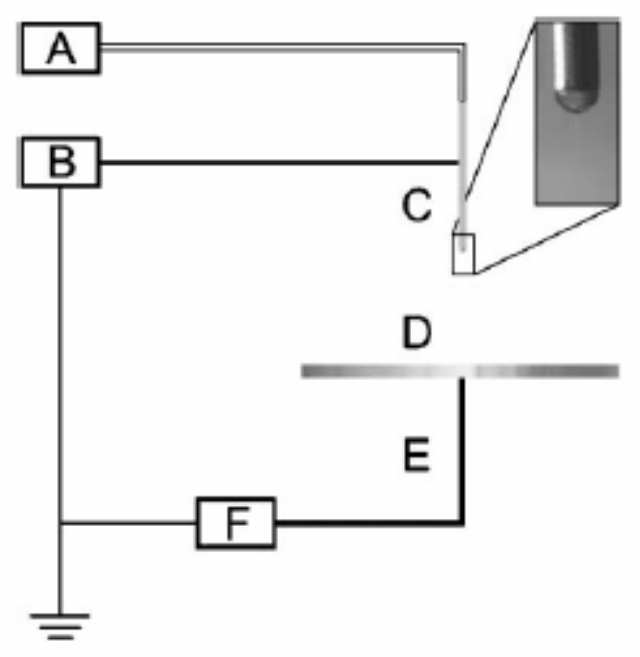

Fig. 1 Schematic representation of the electrospinning apparatus: A. pump; B. high voltage source; C. stainless steel nozzle; D. copper disk $(\mathrm{OD}=15 \mathrm{~cm})$; E. low noise cable; F. picoammeter. 
Since $2002,{ }^{8}$ many reports on the use of sol-gel methods to extend the application of electrospinning toward production of inorganic nanofibres have appeared. Sol-gel Formulations display lower electrical conductivities than conventional ionic solutions of inorganic material precursors, which is necessary for making ultra fine fibres via EHD. The EHD method also arguably more general and less case dependent than self-assembly processes to make submicron solid and hollow fibres and particles, ${ }^{9}$ which often leads researchers to focus more on the processing aspects of the technique than on the underlying chemistry .However, unlike the much larger volume of electrospinning work dealing with the use of dissolved organic polymers, conventional Sol-gel formulations are almost invariably EHD -processed, while condensation and polymerization reactions, such as ones shown below for the well known tetraethyl orthosilicate (TEOS) system, are still occurring:

Hydrolysis:

$\mathrm{Si}\left(\mathrm{OCH}_{2} \mathrm{CH}_{3}\right)_{4}+\mathrm{H}_{2} \mathrm{O} \rightarrow \mathrm{H}_{2} \mathrm{OSi}\left(\mathrm{OCH}_{2} \mathrm{CH}_{3}\right)_{3}+\mathrm{HOCH}_{2} \mathrm{CH}_{3}$

Condensation:

$2 \mathrm{HOSi}\left(\mathrm{OCH}_{2} \mathrm{CH}_{3}\right)_{3} \rightarrow\left(\mathrm{CH}_{3} \mathrm{CH}_{2} \mathrm{O}\right)_{3} \mathrm{SiOSi}\left(\mathrm{OCH}_{2} \mathrm{CH}_{3}\right)_{3}+\mathrm{H}_{2} \mathrm{O}$

The alkoxy product of the second step of this acid-or base-catalyzed system undergoes further condensation, leading to gelation into an amorphous 3-D network. To afford formation of fibres via electrospinning, such sols need to be "aged" to a state close to their gel point. A small fraction of solvent evaporation at the electrified liquid jet stage of the overall process is then all it takes to cross the gel point and the collected fibre mass may then be subject to thermal and gaseous treatments to yield the desirable crystalline state of the fibrous inorganic material, and to remove organic residues and residual solvent.

Thus, the main problem with the processing of a "live" sol is that the time required to obtain gram-quantities of electrospun fibres with a single EHD nozzle is in the order of many hours. Unless the intrinsic reactivity of the sol-gel precursor is modified (e.g., by replacing a fraction of the alkoxy ligands with more stable species such as acetylacetonate or other chelators), or reactions (1) and (2) are judiciously controlled, collection of fibres (or particles, in the case of electrospray) with narrow diameter distributions, and reproducibility of results, are hardly a given. 
Clearly, in this case knowing the starting physical properties of the electrospun liquid and controlling the process variables is not a sufficient measure to control the quality of the fibrous mats obtained via the sol-gel/ EHD technique. The reader is referred to a few excellent sol- gel reviews that cover several aspects of this chemistry, including reactivity control by ligand exchange and reaction kinetics.

Since there is growing interest in electrospun fibres for a variety of applications, and product quality control has now been recognized as a key issue, ${ }^{11}$ we decided to investigate the $\left(\mathrm{CH}_{3} \mathrm{CH}_{2} \mathrm{CH}_{2} \mathrm{O}\right)_{4} \mathrm{Ti} / \mathrm{AcOH} /$ poly (N-vinylpyrrolidone) system in some detail. Based on an extensive search of the open literature, we have found no evidence for sol-gel/EHD studies emphasizing an understanding of the relevant sol-gel chemistry as a means for controlling the quality of electrospun fibre deposits. The model system of choice led to EHD fabrication of titania nanotubes

by Xia and collaborators, ${ }^{9 c}$ who published their research virtually at the same time we published ours on EHD-derived silica nanotubules. ${ }^{9 b}$ Watthanaarun and co-workers ${ }^{9 \mathrm{~d}}$ have also produced titania nanofibres via EHD and a similar PVP/Ti ( $i$-OPr) ${ }_{4}$-based formulation. Our current interest in titania nanofibres also stems from the photo-catalytic properties of this oxide, and our on going effort to produce well-defined nanostructures with different characteristic lengths for future toxicological studies with animal models. ${ }^{12}$ In this paper, we monitor bulk solution properties (e.g., conductivity and viscosity) of representative sols as a function of time, investigate structural features of reactive sols with the aid of in situ infrared spectroscopy, and discuss ways to control the reactivity of this particular titania precursor formulation, which leads to low fibre diameter polydispersity.

\subsection{Experimental}

\subsection{List of abbreviations}

TIP - titanium(IV) isopropoxide, TP - titanium(IV) propoxide, $\mathrm{AcOH}$ - acetic acid, EtOH - ethanol, PVP - poly(N-vinylpyrrolidone).

\subsection{Chemicals and sample preparation}

Titanium (IV) tetrapropoxide (98\%) and poly (N-vinylpyrrolidone) (average molecular weight $\sim 1300000 \mathrm{amu}$ ) were purchased from Aldrich. Titanium (IV) tetraisopropoxide was purchased from Gelest, Inc. (Morrisville, PA). Glacial acetic acid (min. 99.7\%) was purchased from EM Science (Gibbstown, NJ) and anhydrous ethanol (.99.5\%) was purchased from 
McCormick Distilling Co., Inc. (Weston, MO). Cyclohexane (Chempure ${ }^{\mathrm{TM}}$ Brand, non-volatile matter 0.0005\% max.) was purchased from Curtin Matheson Scientific Inc. (Houston, TX).

In a typical procedure, $600.0 \mathrm{mg}$ of PVP was dissolved in a solution containing $17.2 \mathrm{ml}$ of $\mathrm{EtOH}$ and $0.8 \mathrm{~mL}$ of $\mathrm{AcOH}$. The resulting suspension was kept in a capped glass bottle with a Teflon $^{\text {TM }}$-lined PE seal, and magnetically stirred until complete dissolution of the polymer. Subsequently, $2.0 \mathrm{~mL}$ of titanium alkoxide (TP or TIP) was added to the solution using a plastic disposable pipette (it was found that disposing of TP is facilitated when a plastic pipette is used instead of a glass one). For comparing the overall stability of our formulations with previously reported data, the exact sol-gel-based fibre-producing recipe from ref.13 was used in some experiments. In general, one key difference between our approach and the sol preparation protocol in ref.13 is the $\mathrm{Ti} / \mathrm{AcOH}$ molar ratio, which in their case was 1: 10, compared to the 1: 1, 1:2 and 1: 4 ratios adopted in the present studies. In addition, their procedure was based on preparation of two separate solutions ( $\mathrm{TIP} / \mathrm{AcOH}$ in $\mathrm{EtOH}$, and then PVP in EtOH) and mixing them at a later stage. A very similar procedure could be found in the work of Yoshida et al., ${ }^{14}$ the only difference being that ethanol was used for electrospinning work, instead of 2-propanol.During the transfer of moisture-sensitive TP and TIP, attention was paid to protect these chemicals from direct contact with air by using an argon atmosphere.

\subsection{Viscosity and electrical conductivity measurements}

The viscosity of solutions was measured with a Brookfield DV-II+ programmable rotating drum type viscometer (Brookfield Engineering Lab. Inc., Middleboro, MA) at $25{ }^{\circ} \mathrm{C}$ with an SC-18 spindle. Several rotational speeds were used.

The electrical conductivity was determined with the aid of a digital conductivity meter with a platinum dip cell (Traceable ${ }^{\mathrm{TM}}$, VWR Scientific, and West Chester, PA). The cell was dipped in the solution, and was then gently moved up and down a few times to remove any air bubbles stuck to its surface. The measurement was performed in the continuous mode with the electrode inserted into the capped bottle filled with argon. To prevent creation of an electric double layer on the interface between electrode and measured solution, stirring with a magnetic stir bar was applied. 


\subsection{UV-VIS and FTIR spectroscopy}

UV-VIS spectra were collected using a Beckman DU-640 spectrophotometer. In a typical experiment, $1 \mathrm{ml}$ of solution was transferred into the cuvette with the aid of a Pasteur pipette. The cell was made of quartz ('Q $Q$ ' type), and had a $10 \mathrm{~mm}$ optical path. Spectra were recorded in the range 200$800 \mathrm{~nm}$ with a resolution of $1 \mathrm{~nm}$, with anhydrous ethanol used as a blank.

FTIR spectra were recorded using a Nicolet $20 \mathrm{XB}$ spectrometer, in the range $4000-500 \mathrm{~cm}^{-1}$, and typically with a spectral resolution of $2 \mathrm{~cm}^{-1}$. A sealed liquid cell Model SL-3 with ZnSe windows with circular aperture and $0.015 \mathrm{~mm}$ path length was purchased from International Crystal Laboratories. Spectra were collected in the transmittance mode, using the empty cell as background. In the next step, all spectra were normalized and converted to absorbance units. Then, the solvent (EtOH) spectrum (corrected for concentration of particular species present in the solution) was subtracted from those of actual runs. Spectra of either ethanol solutions of pure ingredients used in the preparation of titania sols, or titania sol samples were collected. In some cases, for the sake of clarity, solutions of titanium alkoxides in cyclohexane were examined. After careful analysis, it appeared that only bands located below $2000 \mathrm{~cm}^{-1}$ play an important role in the detection of relevant changes in the structure of sols as a function of time.

\subsection{Electrospinning and SEM microscopy}

The titania sol-gel precursor solution was loaded into a Hamilton series 1000 gastight syringe (model 1001, $1 \mathrm{ml}$ ) using a 19 gauge metal needle. The syringe was placed on the syringe holder of a digital syringe pump (Cole-Parmer 7490000, Vernon Hills, IL), and a liquid delivery plastic tube (ca. $25 \mathrm{~cm}$ long) was attached by connecting it to the syringe through an appropriate fitting. The opposite end of the tubing was attached to a $5 \mathrm{~cm}$ long piece of 19 gauge stainless steel tubing, which served as an electrospinning nozzle. A voltage in the range 5-10 kV, with $1 \mathrm{kV}$ steps, (Gamma High Voltage Research ES30P-5W/PRG, Ormond Beach, FL) was applied to the nozzle, and after 5 minutes of “electrostatic equilibration”, experiments were carried out.

During a typical experiment, flow rates in the range $0.2-0.5 \mathrm{ml} \mathrm{h}^{-1}$ were used, and collection time was limited to 10-15 minutes. The distance between the tip of the nozzle and the collector plate was fixed to $5 \mathrm{~cm}$. Fibres were collected over the surface of a piece of aluminium foil tightly wrapped around the copper disk $(\mathrm{OD}=15 \mathrm{~cm})$ serving as the collector electrode (Fig. 1). 
The current carried by the electrospun fibres was recorded using a Keithley picoammeter (Model 6485), which was connected in series to the collector plate via a low-noise coaxial cable (Model 4801) with male BNC connectors.

After the electrospinning experiments were performed, the "as-collected" fibres were left for 24 hours on the aluminium foil, and hydrolysis of TP was assumed to be complete. SEM imaging experiments were performed with the aid of a Hitachi S-3000N microscope, using an accelerating voltage of $15 \mathrm{kV}$. Before samples were placed in the microscope chamber, they had to be coated with metallic chromium for 2 minutes to boost their electrical conductivity, and thus improve their SEM contrast. Statistical analysis of the acquired SEM images comprised evaluation of the diameter of many fibres, and a set of 150 measurements was assumed to be representative for a particular sample.

\subsection{Results and discussion}

\subsection{Rationale}

It occurred to us, after observing that a mixture of TP, glacial acetic acid, and 200-proof ethanol remained clear for over a month and yielded propyl acetate in solution, that either a Ti ligand exchange mechanism or a very slow non-hydrolytic sol- gel reaction pathway was responsible for the long-term EHD process ability of this solution. Conductivity and viscosity changed very slowly with time. Addition of PVP, a common plasticizer, aids in the fibre formation process in general because longchain polymer additives tend to lower surface tension and increase viscosity. Note that besides solvent evaporation during the time-of-flight from nozzle to collector electrode, ambient moisture would react with the ultra fine charged liquid jets, leading to further hydrolysis of our formulation. However, the chemical consequences of adding PVP prior to EHD processing also deserved some attention.

The analysis of complexation of TP by acetic acid has been based on the following assumptions: first, ligand exchange reactions between solvent molecules were neglected, despite that there are reports

in the literature suggesting such a possibility. ${ }^{15}$ In ref. 15, ligand exchange in the system of TIP with pure $\mathrm{EtOH}$ was discussed, and it was found that there was only one isopropyl group exchanged per molecule of ethanol. In our case, a much stronger complexation agent than EtOH is obviously present, so the aforementioned assumption can be easily justified at least during the early stages of reaction (first few hours). Second, in the case of diluted titanium alkoxide solutions there is the unlikely possibility of esterification taking place within the reaction time span monitored in this study. It was observed that after three weeks homogeneously generated water (as a by-product of the esterification reaction) was the source of precipitation in the case of $\mathrm{TP} / \mathrm{AcOH}$ 


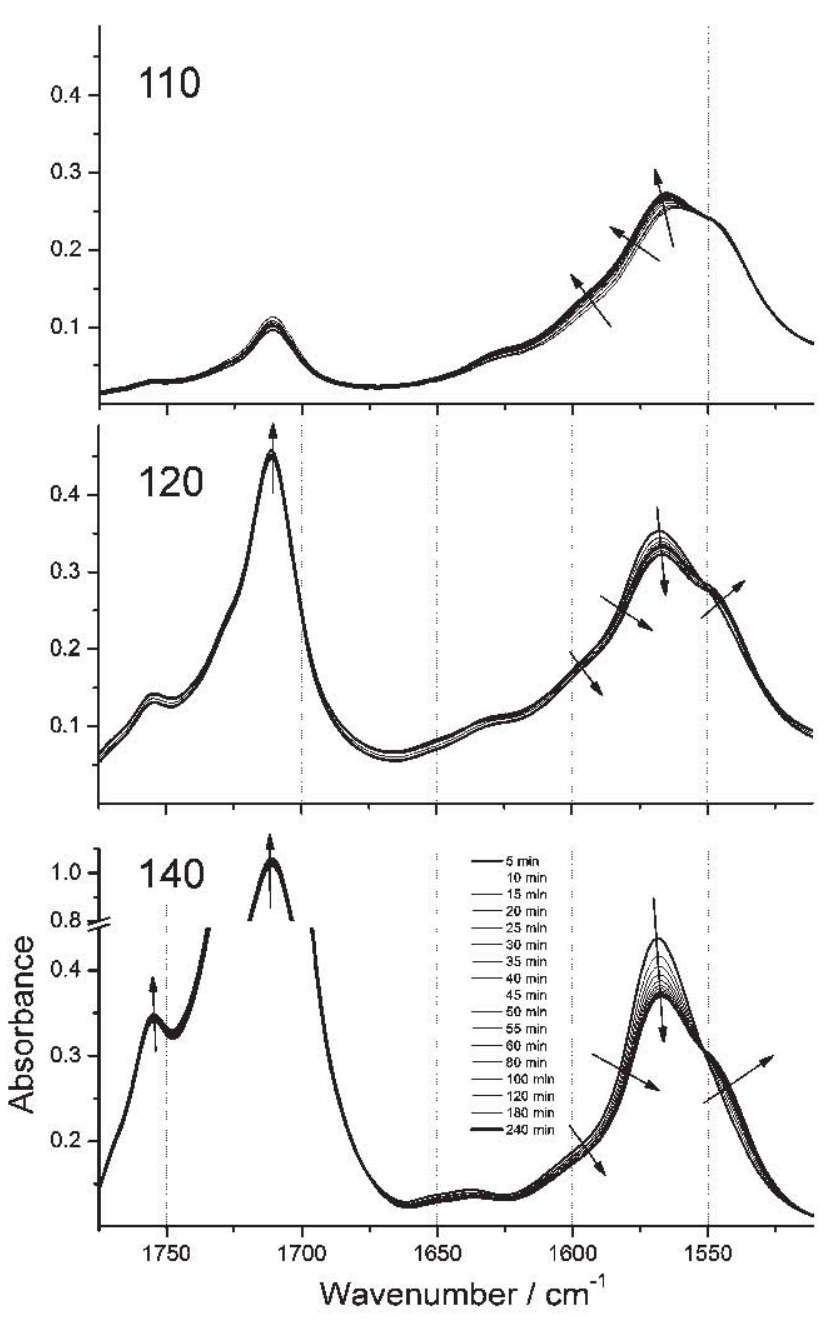

Fig. 2 Evolution of the TP/AcOH mixtures (solvent-subtracted), as monitored by IR in the $1510-1770 \mathrm{~cm}^{-1}$ region.

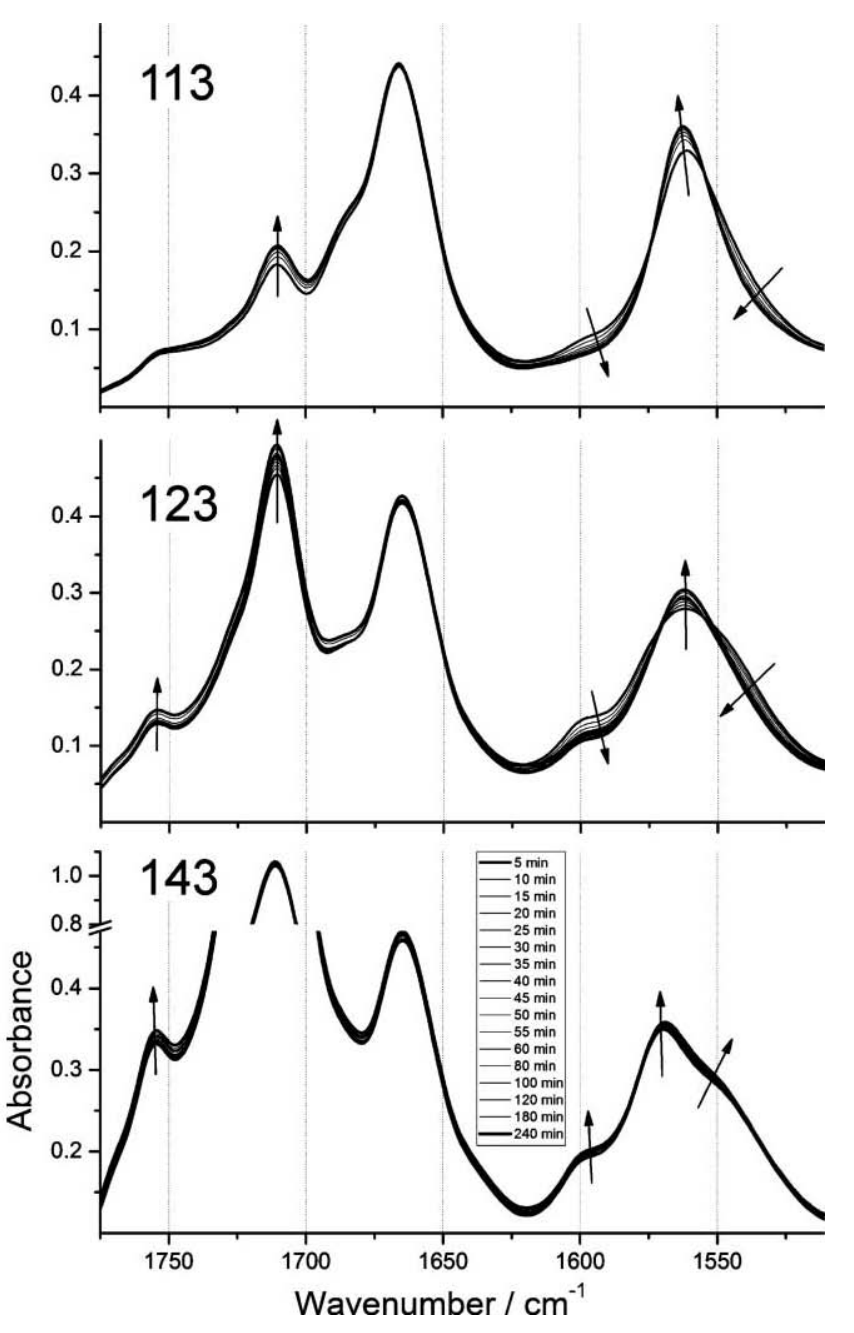

Fig. 3 Evolution of the TP/AcOH/PVP mixtures, as monitored by IR (solvent-subtracted) in the $1510-1770 \mathrm{~cm}^{-1}$ region.

mixtures with the highest molar ratios (denoted as 140, "0" standing for no PVP). This observation is in line with studies where the pure chemicals were used, i.e. within a few hours from the beginning of reaction esters had not been detected. ${ }^{16}$ Low ester concentrations had been detected after extended times $(6.5 \mathrm{~h}),{ }^{17 \mathrm{a}}$ or when the esterification reaction rate had been intentionally increased by heating. ${ }^{17 \mathrm{~b}}$ Interestingly, in the case of the TP/AcOH/PVP mixture (143 sample) a white precipitate appeared after a much shorter time (a few hours), and propyl acetate was also detected by FTIR. Propyl acetate has strong bands at $1744 \mathrm{~cm}^{-1}$ and $1720 \mathrm{~cm}^{-1}$, which differ significantly from those of free acetic acid in this region. This suggests propanol molecules are present in these solutions in larger amounts than in the polymer-free system. 
The potential AcOH/PVP interactions in this ternary system also deserve some analysis. Studying the infrared spectrum of solutions of polyphosphoric acid/PVP in methanol, Bozkurt and Meyer ${ }^{19}$ showed that protonation occurs primarily on the CLO group of the vinylpyrrolidone moiety, instead of the nitrogen atom. The sharp CLO stretching signal from PVP around $1670 \mathrm{~cm}^{-1}$ becomes significantly broader (to about a 100-200 $\mathrm{cm}^{-1} \mathrm{FWHM}$ ), and shifts toward lower frequencies in the presence of PPA. From Figs. 2, 3 and 4, it is clear that acetic acid is unable to cause the same changes on the $1670 \mathrm{~cm}^{-1}$ PVP IR band in the presence of the Ti alkoxide, despite the fact that PVP-induced disruption of the $\mathrm{TP} / \mathrm{AcOH}$ complex is evident from the changes in the $v_{\mathrm{as}}(\mathrm{COO})$ region. The $\mathrm{AcOH} /$ vinylpyrrolidone moiety ratio in our case was 2.5: 1 (for the solutions with $\mathrm{TP} / \mathrm{AcOH}$ molar ratio equal to $1: 2$, which would have more than justified some level of protonation in light of mere stoichiometric arguments. The convoluted $1100-1300 \mathrm{~cm}^{-1} \mathrm{C}-\mathrm{N}$ and $\mathrm{C}-\mathrm{O}$ stretching regions (not shown) also do not display any dramatic evidence for acetic acid/PVP interaction.

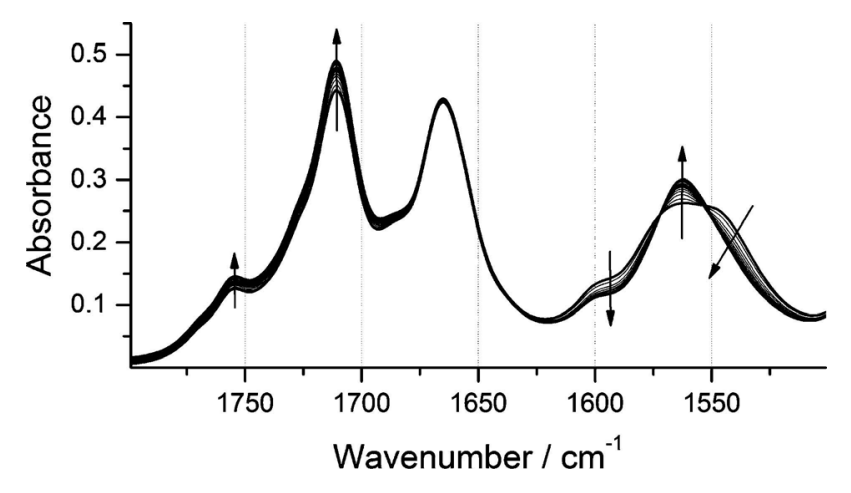

Fig. 4 Evolution of the TP/AcOH/PVP mixture prepared by the two-pot method, as monitored by IR (solvent-subtracted) in the 1510$1770 \mathrm{~cm}^{-1}$ region. 


\subsection{Titanium(IV) propoxide and acetic acid interactions - diluted medium scenario}

The first possibility in liquid media with only trace amounts of water and no PVP is attack by acetic acid of the TP alkoxide leading to the three coordination structures proposed by Perrin et al. ${ }^{16}$ Indeed, the work of these authors suggests that acetic acid/complex formation is rather easily tracked by following the $v_{\text {as }}(\mathrm{COO})$ region (Fig. 5). In Fig. 2, the evolution of the $1510-1770 \mathrm{~cm}^{-1}$ IR region of the TP/AcOH mixture as a function of time is shown. For brevity, detailed analysis of the $v_{\text {as }}$ (COO) 1330-1510 $\mathrm{cm}^{-1}$ region (which actually requires deconvolution of other minor IR bands ${ }^{16}$ ) was not performed. Such analysis would provide the same information as that derived from interpretation of the $v_{\mathrm{as}}(\mathrm{COO})$ IR bands. Similarly, the $1700-1800 \mathrm{~cm}^{-1}$ CLO stretching region reveals little new information in terms of propyl acetate ester formation.

At the chosen $\mathrm{TP} / \mathrm{AcOH}$ ratios, Fig. 2 indicates that the chelating bidentate structure is predominantly stabilized within a few hours (1540-1550 $\mathrm{cm}^{-1}$ shoulder), with a concomitant decrease in the bridging complex $v_{\text {as }}(\mathrm{COO})$ signal at the centre of this band group. There are thus two modes of complexation of TP by acetic acid that could ultimately be discerned in light of electrical conductivity and vibrational spectroscopy data: associative (structures $\mathbf{1}$ and 2, Fig. 5) and dissociative (structure 3, Fig. 5) complexation pathways are possible. Complexes 1 and 2 increase the conductivity of the solution because on creation of these species, charged complexes and one proton are generated (recombination of propoxy ligands with free protons is the main source of conductivity decay of solutions in our case). Creation of complexes characterized by structure $\mathbf{3}$ (dissociative mode) is generally accepted in the scientific literature. ${ }^{20}$ The propoxy ligands detached from the alkoxide molecule by this reaction pathway are expected to recombine stoichiometrically with protons released by the acetic acid on their ligation to the Ti centres. The net effect of this reaction on the electrical conductivity of the solution is expected to be negligible in comparison with that of formation of complexes $\mathbf{1}$ and $\mathbf{2}$ in Fig. 5. On the other hand, in the dissociative complexation mode, acetate ligands are bound to titanium atoms less strongly than in the associative cases because they are stabilized by propoxide groups located at the bridging positions, so certain infrared bands are located at higher wavenumbers (e.g., $1590 \mathrm{~cm}^{-1}$ signal). 
Shifts in the equilibrium between the bidentate (1) and bridging (2) complexes are observed by changing the molar ratio of TP to $\mathrm{AcOH}$ from $1: 1$ to $1: 4$. As expected, with a molar ratio of $1: 1$ the bidentate complex is dominant (Fig. 6, sol labelled as 110), but the overall concentration of charged species in such low acetic acid concentrations is relatively small compared to the acetic acidrich cases. Nevertheless, it is worth noting that there still is free acetic acid in solution in the TP/AcOH $1: 1$ case (Fig. 2, sample 110, band at $1710 \mathrm{~cm}^{21}$ ). With increasing acetic acid content, the overall amount of both

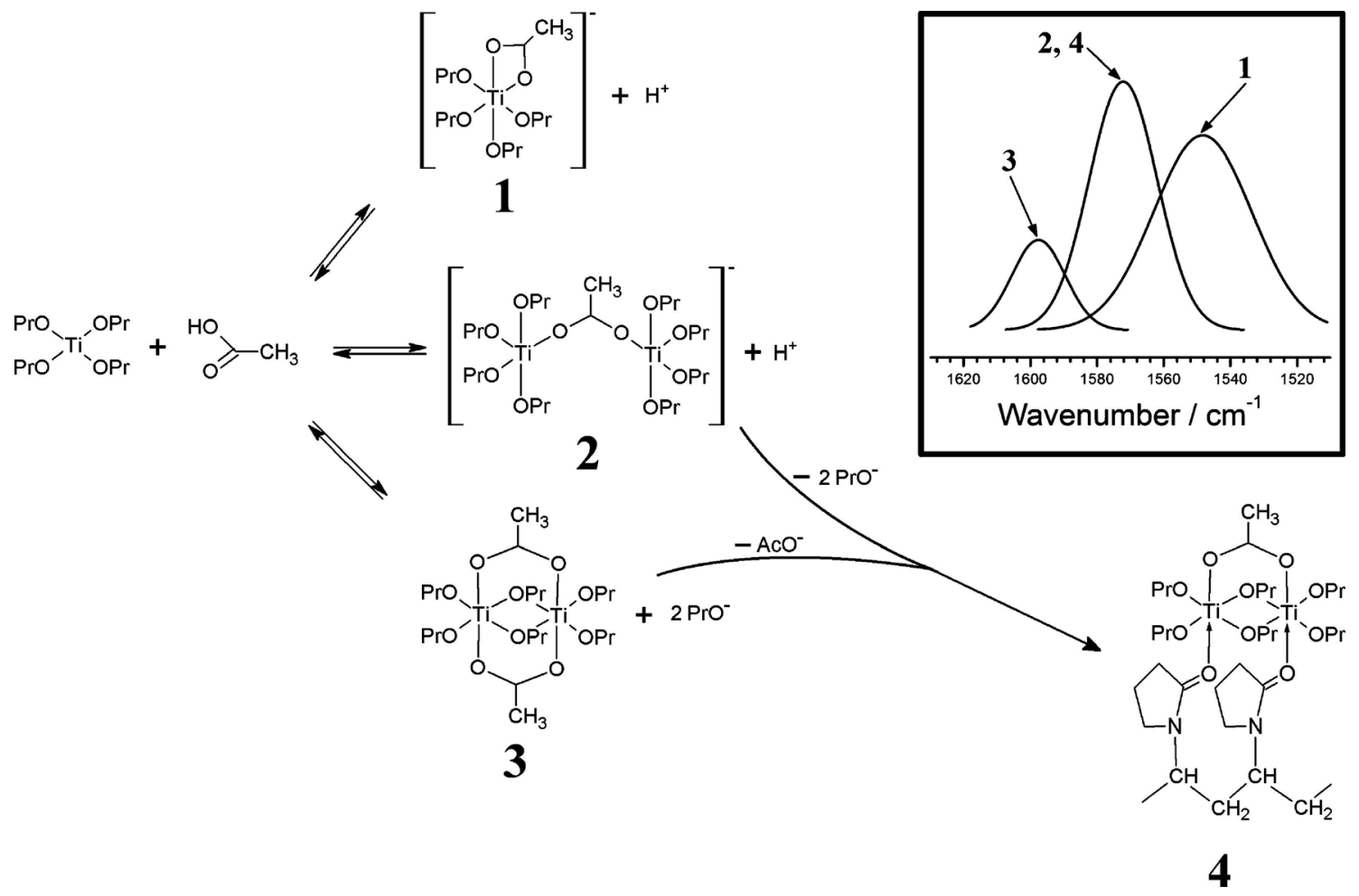

Fig. 5 Proposed reaction pathway, coordination modes of acetic acid with $\mathrm{Ti}$ alkoxides, and infrared band assignments in the $\mathrm{v}_{\mathrm{as}}(\mathrm{COO})$ region from a deconvoluted spectrum, according to refs. 16 and 18. 

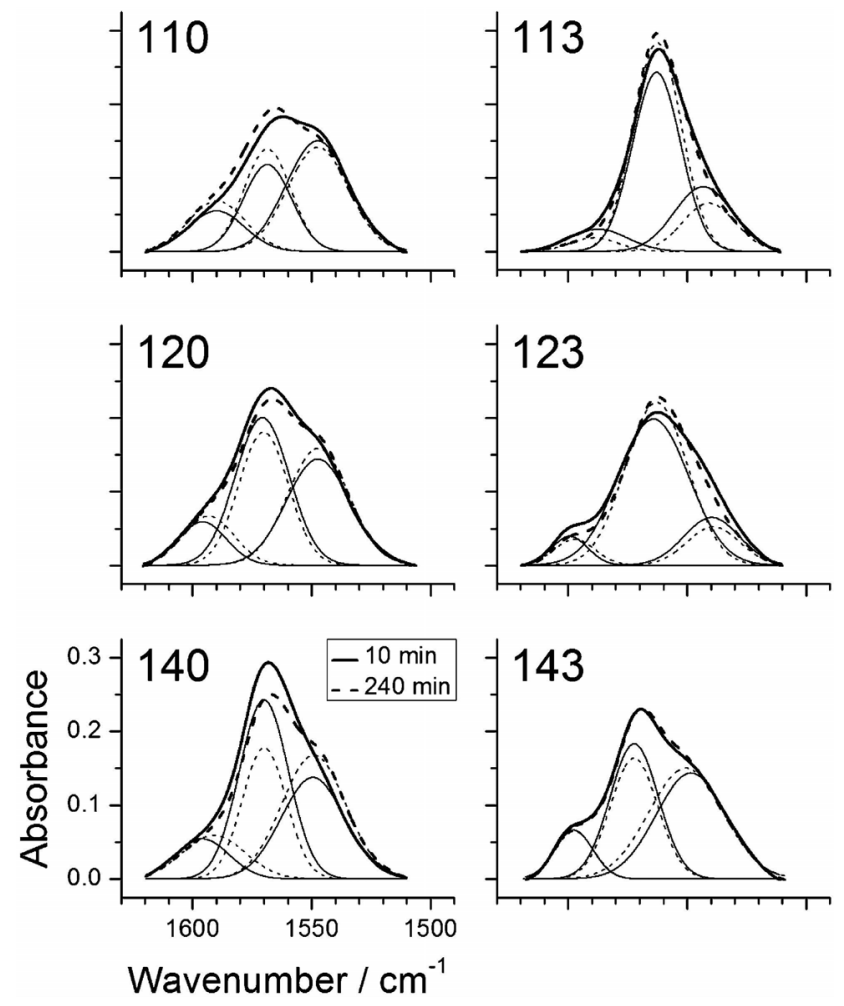

Fig. 6 Evolution of the deconvoluted bands in the $v_{\mathrm{as}}(\mathrm{COO})$ region as a function of time for the TP/AcOH and TP/AcOH/PVP mixtures obtained by mixing all ingredients at once.

types of complexes increases, and the equilibrium is shifted toward the bridging form (Fig. 6, sols 120 and 140).

Within the quality of the IR signal achievable in the $500-700 \mathrm{~cm}^{-1}$ range, the $\mathrm{n}(\mathrm{Ti}-\mathrm{O})$ band also shows evidence for $\mathrm{AcOH} / \mathrm{Ti}$ complex formation. Changes in intensity as a function of acetic acid concentration of the 611 and $583 \mathrm{~cm}^{-1}$ bands support the idea that association among Ti species in solution exists (see the IR traces at $\mathrm{t}=5$ and $240 \mathrm{~min}$, inset of Fig. 7). ${ }^{-1}$ After one hour, profiles of recorded spectra in this region clearly show very similar modifications to the titanium(IV) coordination sphere by acetic acid, independent of the molar ratios used (Fig. 7, left). This supports the general conclusion that in the case of diluted solutions of titanium(IV) alkoxides modified by acetic acid the overall degree of association among titanium complexes is low. 
The temporal evolution of these solutions was also investigated: The bridging complex 2 is generated first, and is then partially converted to the bidentate structure 1 (Fig. 6, left panels). Differences in the relative population of each complex as equilibrium is approached results from differences in the initial acetic acid concentration. This provides an explanation for the slight differences in conductivity among the different $\mathrm{TP} / \mathrm{AcOH}$ mixtures (Fig. 8).

\subsection{Sol-gel chemistry of titanium(IV) alkoxide/AcOH as modified by PVP}

The most pronounced effect of addition of PVP to the AcOH/ TP sol is a remarkable increase in the viscosity of the system, and the presence of acetic acid then facilitates interaction of titania species with the polymer. We observed that interactions of TP and PVP dissolved in ethanol without acetic acid are rather weak in the context of modification of the rheological properties of the system. However, the viscosity of this mixture is higher than that of the solution of an equivalent amount of pure polymer in ethanol (Fig. 9). The interaction of PVP with Ti species in the presence of acetic acid also causes the appearance of colour. None of the other possible combinations of chemicals used in this study gives such a strong absorption band in the visible region (Fig. 10). Ligand-to-metal charge transfer (LMCT) from the pyrrolidone groups into unoccupied d-orbitals of titanium(IV) are expected to be responsible for this UV absorption edge, in analogy to those found in metallocenes (Cp)2Ti(IV)X2, where Cp = cyclopentadienyl ( $\left.{ }^{5}-\mathrm{C} 5 \mathrm{H} 5\right), \mathrm{X}=$ halide or pseudo-halide. ${ }^{22}$ Among the titanium(IV) acetate complexes existing in the solution, only those having a bridging acetate group can be attached to the polymer chain through a typical coordinative bond. Note that infrared (acetate) signals from structures 2 and 4 would strongly overlap, and an increase in PVP concentration shows
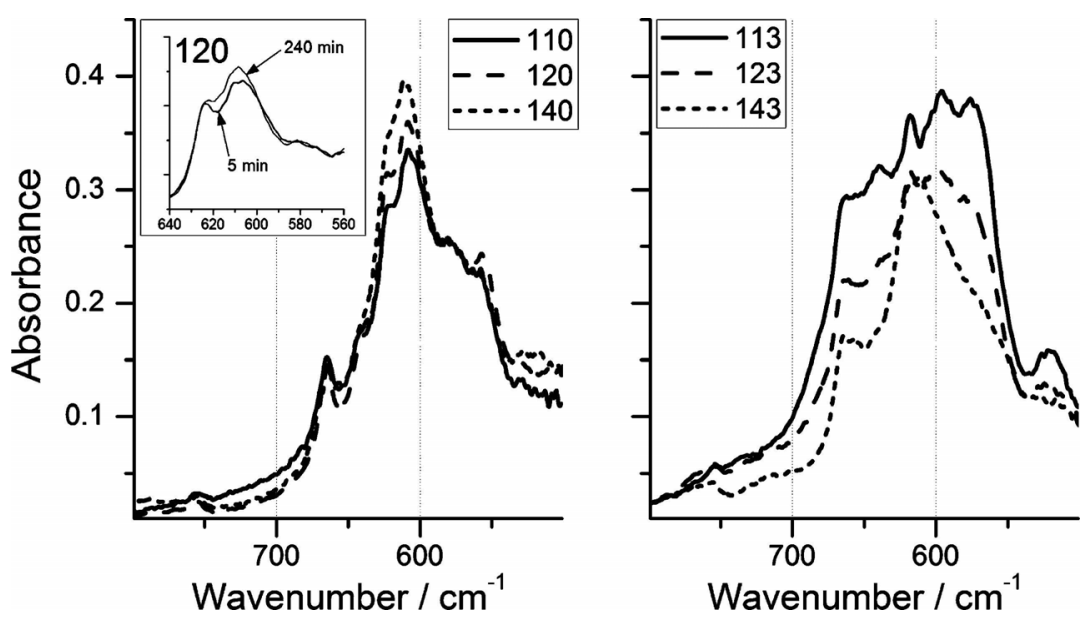

Fig. 7 FTIR spectra of the $v(\mathrm{Ti}-\mathrm{O})$ region $\left(500-800 \mathrm{~cm}^{-1}\right)$ after 60 minutes of reaction as a function of different TP/AcOH and TP/AcOH/PVP molar ratios (solvent-subtracted). Inset: temporal evolution of the $v(\mathrm{Ti}-\mathrm{O})$ bands (sample 120). 


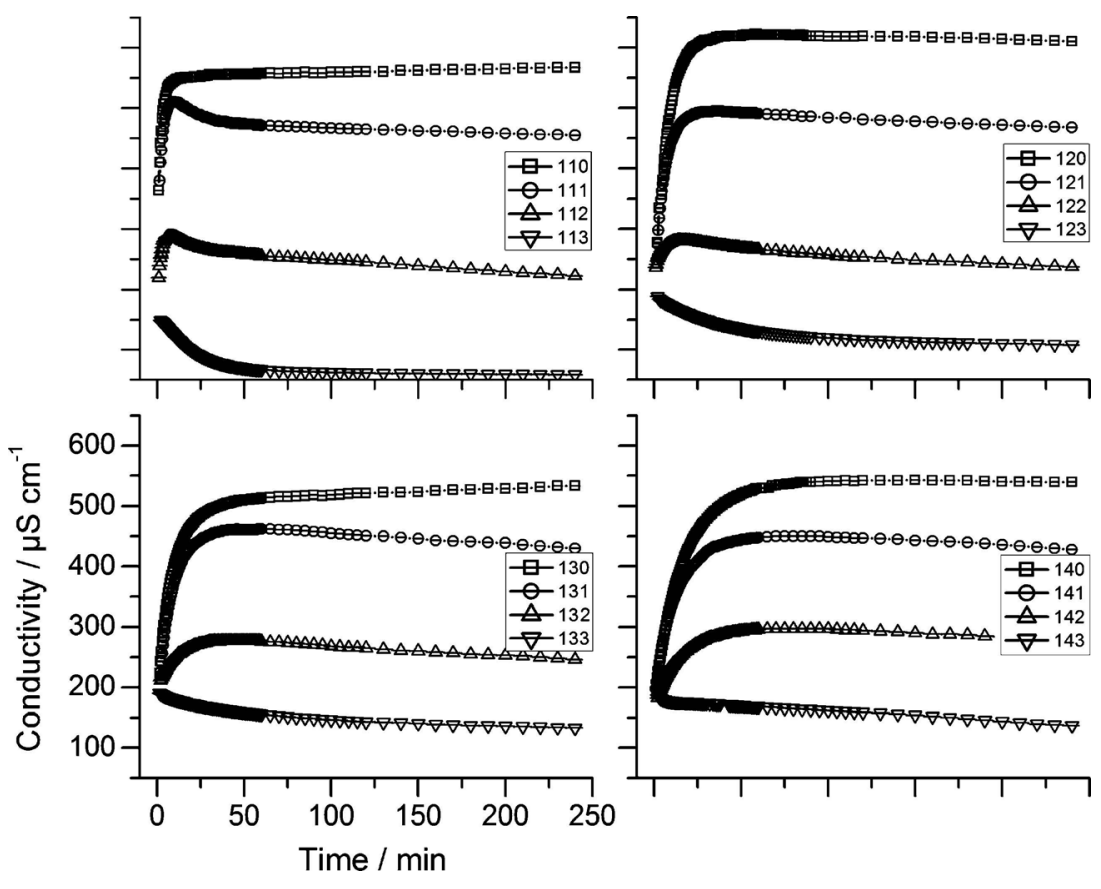

Fig. 8 Electrical conductivity as a function of time. The T/AcOH/PVP ratios are indicated directly in the figure. The first two digits represent the $\mathrm{Ti} / \mathrm{AcOH}$ molar ratio. The third digit gives the w $\%$ of PVP in solution.
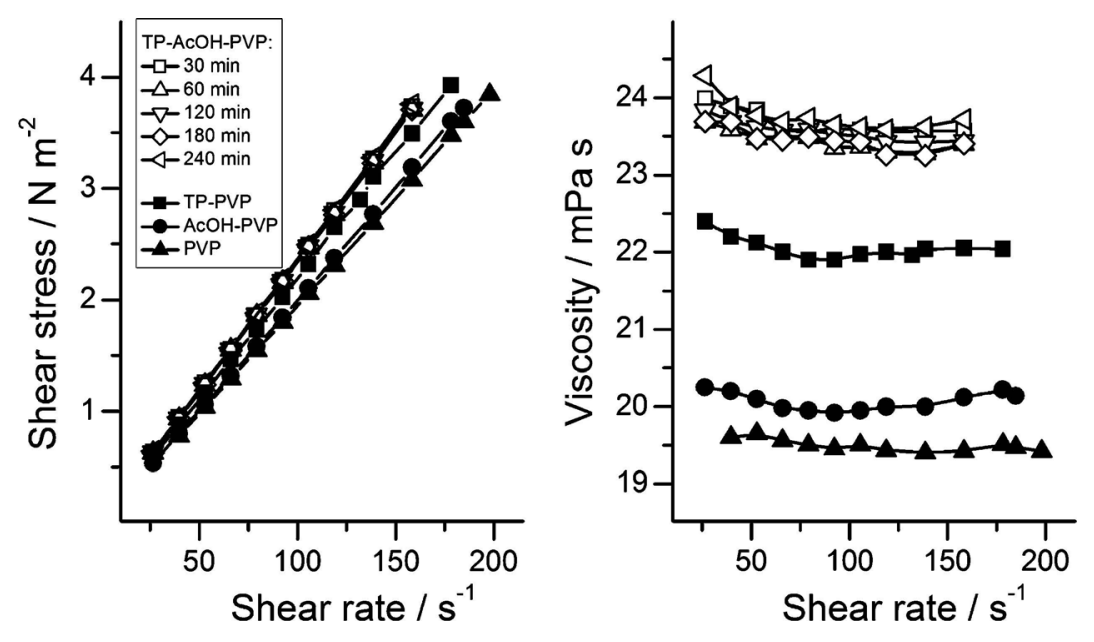

Fig. 9 Representative viscosity data for Ti:AcOH:PVP sols and the pure ingredients dissolved in ethanol. 

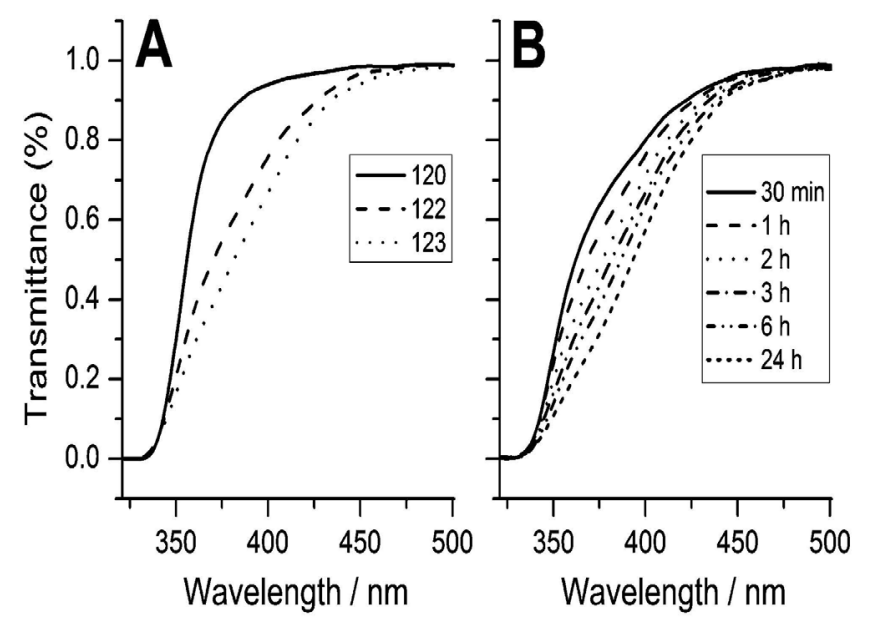

Fig. 10 Normalized UV-VIS spectra of the TP-AcOH-PVP system (molar ratio $\mathrm{TP}: \mathrm{AcOH}=1: 2$ ): A) Influence of polymer addition, B) Sample 123.

that this is indeed the case (Fig. 5 and Fig. 6). The bridging acetate group, shown sitting "on top" of the complex in Fig. 5, structure 4, is expected to have nearly the same infrared spectral characteristics as those of the free bridging complex (2, Fig. 5). During this process the displaced ligands are expected to recombine with free protons, leading to a decrease in electrical conductivity. Note also how the intensity of the $\mathrm{n}$ (CLO) band of acetic acid increases with time (Fig. 3).

Besides the observed impact of PVP on the physical properties of these solutions, it is particularly informative to understand how PVP affects the Ti/AcOH complexation chemistry. Fig. 6, shows how PVP disrupts or reverses formation of the bridging and chelating bidentate $\mathrm{AcOH} / \mathrm{TP}$ complexes, as evidenced by a gradual disappearance of the 1590 and $1540 \mathrm{~cm}^{-1}$ shoulders around the $v_{\text {as }}$ (COO) main peak of the bridging complex. 
The band located at $1563 \mathrm{~cm}^{-1}$ shifts toward higher wavenumbers with an increasing $\mathrm{TP} / \mathrm{AcOH}$ molar ratio. This band is the dominant spectral feature of the $v_{\mathrm{as}}$ (COO) region in the case of the PVP-rich 113 sample (Fig. 3 and Fig. 6), and the relatively large concentrations of such a non-ionic complex are also responsible for a severe decrease in conductivity. This is not what it is observed when larger amounts of acetic acid are present (Fig. 8). Thus, the charge-bearing $\mathrm{Ti}^{4+} / \mathrm{AcOH}$ complexes (especially bridging ones) are effectively attacked by PVP. A higher acetic acid content helps keep the conductivity of the solution relatively high (at least twice as high as that displayed by the 113 sample), by shifting the equilibrium toward the bidentate complexes (1, Fig. 5). The latter cannot be attached to the polymer in one step, but must first be converted into bridging species.

TP was also allowed to react with $\mathrm{AcOH}$ for one hour prior to mixing with the PVP/EtOH solution (Fig. 4). In Fig. 3, the same IR region is shown for the "one-pot" approach, in which PVP, AcOH, TP and ethanol are mixed all at once. Note that for short reaction times the bridging and chelating bidentate signals are evident, but PVP slowly brings the system to a similar stable state as that observed for the "two-pot" formulation. This was also confirmed by conductivity measurements (Fig. 11). One difference that was observed between these two modes of sol preparation is that in the case of the twopot approach, conductivity decays more rapidly after mixing than in the one-pot case. Since the PVP-free system reaches equilibrium after about one hour (see sol 120 in Fig. 8), the TP/AcOH complexes are all formed and ready to interact with PVP on mixing. By contrast, in the one-pot case they first must be generated in situ.

\subsection{EHD implications of TP/AcOH/PVP Chemical Equilibria}

The overall stabilization of the chemical state of the TP/AcOH/PVP system after about one hour is what makes it especially suitable for reproducible EHD processing. Without a slow-changing conductivity, the chances for continuous EHD processing of a metal oxide precursor formulation into a fibrous mat with a narrow fibre diameter distribution are practically non-existent. To a first approximation, these TP/AcOH/PVP formulations are also Newtonian fluids (see Fig. 9), a fact that permits adoption of simple

models to predict the terminal jet diameter (and consequently, fibre diameter), ${ }^{7 a}$ over more complex ones. 

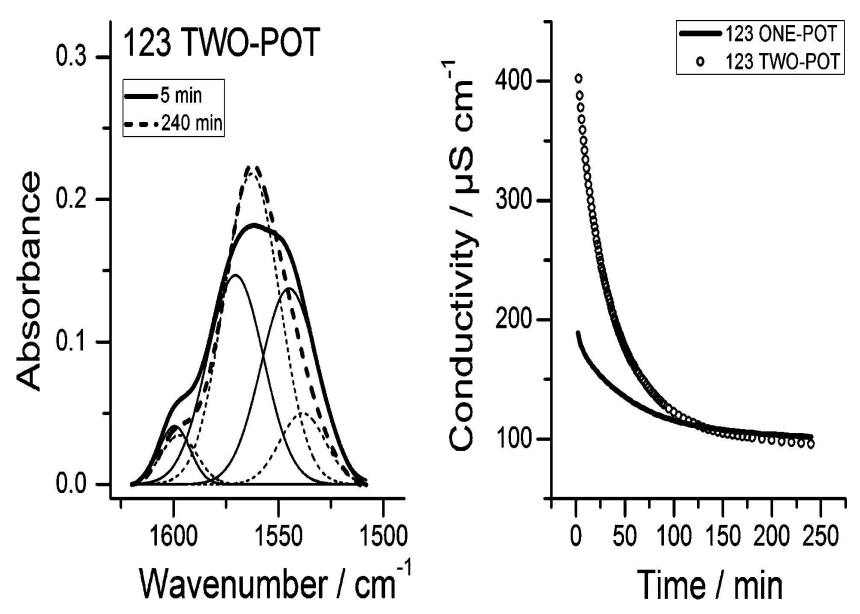

Fig. 11 Deconvoluted bands in the $v_{\text {as }}(\mathrm{COO})$ region of the mixture prepared using the two-pot method (left section) and conductivity differences between the two sol preparation methods (right section).

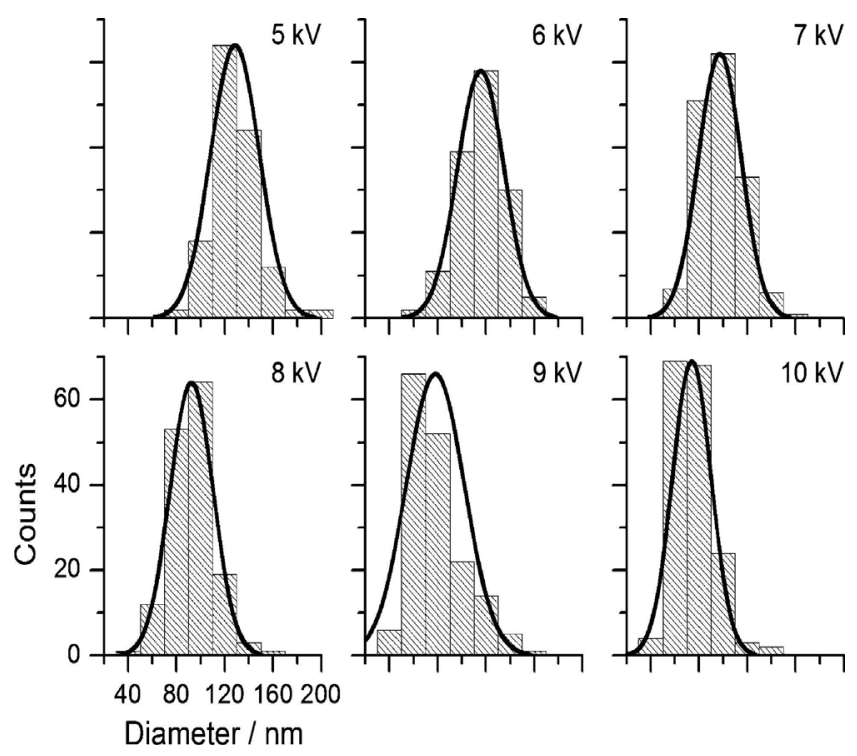

Fig. 12 Fibre diameter distribution as a function of applied voltage.

Flow rate: $0.2 \mathrm{~mL} \mathrm{~h}^{-1}$. 


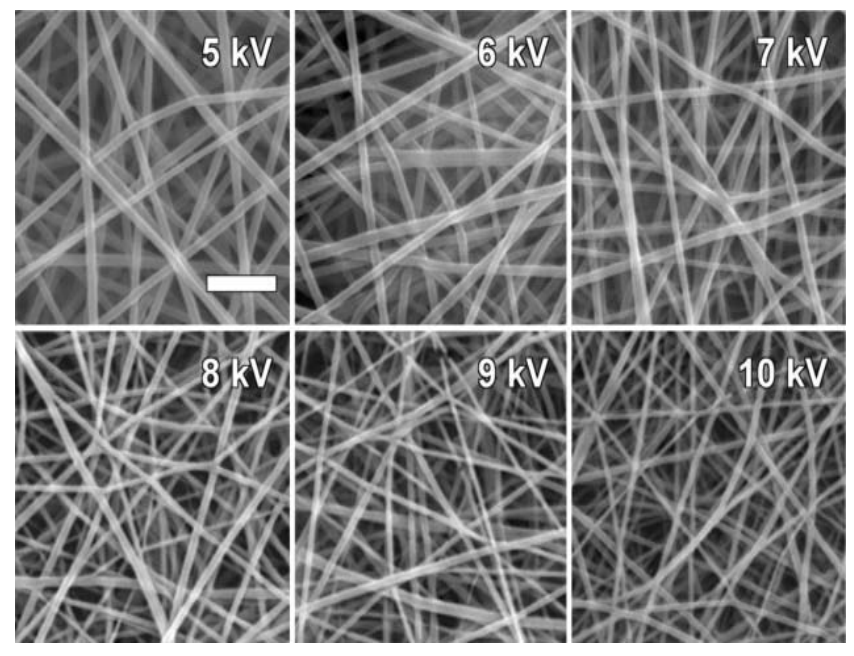

Fig. 13 SEM images of $\mathrm{TiO}_{2} / \mathrm{PVP}$ composites produced under different applied voltages and flow rate $0.2 \mathrm{~mL} \mathrm{~h}^{-1}$. Scale bar: $1 \mu \mathrm{m}$.

As predicted by EHD jet thinning theory, ${ }^{24}$ analysis of scanning electron microscopy data reveals that fibre diameter is inversely proportional to electric field strength, in our case controlled by voltage changes at a fixed electrode separation (Fig. 12). From these results, it is apparent that the stability of these solutions translates into fibre diameter distributions that appear to be quite narrow (Fig. 13) relative to what is typically obtained by EHD processing of sol-gel formulations.

\subsection{Conclusions}

Better understanding of the underlying chemical processes is necessary to prepare sols that afford reliable processing into electrospun fibres. In this particular ternary system, it is clear that the physical properties of these sols change slowly over time because complexation equilibria in a water-deficient liquid medium retard significantly the oligomerisation of dissolved $\mathrm{Ti}$ species. Solid fibres are thus formed by mere precipitation of these species by solvent evaporation during the time-of-flight from electrode to electrode, and further cross-linking and calcinations of as-deposited fibres on heating ultimately provides the desired titania phase

Acetic acid helps maintain conductivity at the levels needed for successful EHD processing of these ternary sols into smooth fibres with rather narrow diameter distributions. This is achieved by creation of charged complexes with TP, and by an 
obvious release of protons, even though in a dry alcoholic solution acetic acid is so weak an electrolyte that it is known to remain practically in its non-dissociated form. FTIR analysis of the acetic acid $v_{\mathrm{as}}(\mathrm{COO})$ region reveals two types of $\mathrm{TP} / \mathrm{AcOH}$ complexation, namely associative and dissociative. The former complexes raise the conductivity of these solutions, while the latter plays an important role in facilitating the interaction between Ti species and PVP.

Addition of a polymer such as PVP is expected to facilitate elongation of an electrified liquid jet to make fibres before the onset of Rayleigh instabilities, by desirable changes in the viscosity and surface tension of the sol. However, PVP is also shown to be chemically active as a ligand, and as an agent that also controls the complexation kinetics and sol-gel chemistry of the system. We believe this is likely a general conclusion that may apply to similar non-hydrolytic sols when the solution property-modifying polymer has chemically active groups, and to some extent it may also apply to hydrolytic cases involving labile ligands. Regardless of the order and mode in which these three components are mixed, they reach the same chemically stable state after a few hours of reaction. This solution stability, coupled with favourable physical property values of the sols, afford production of well-defined ultrafine fibres via EHD.

\subsection{Acknowledgements}

Funding from the Nebraska Research Initiative and the National Institutes of Health (1 R43 ES013367-01 A1) is gratefully acknowledged. 


\section{References}

1. Lord Rayleigh, Philos. Mag., 1882, 44, 184.

2. J. Zeleny, Phys. Rev., 1917, 10, 1.

3 .A. Formhals, U.S. Pat., 1975 504, 1934; A. Formhals, U.S Pat., 2160 962, 1939; A.

Formhals, U.S. Pat., 2187 306, 1940; A. Formhals, U.S. Pat., 2323 025, 1943; A.

Formhals, U.S. Pat., 2349 950, 1944.

4(a). J. Doshi and D. H. Reneker, J. Electrost., 1995, 35, 151;

(b) D. H. Reneker and I. Chun, Nanotechnology, 1996, 7, 216.

5 .G. I. Taylor, J. Fluid Mech., 1965, 22,1.

6. M. Cloupeau and B. Prunet-Foch, J. Aerosol Sci., 1994, 25, 1021.

7(a) S. V. Fridrikh, J. H. Yu, M. P. Brenner and G. C. Rutledge,Phys. Rev. Lett., 2003, 90, 144502; (b) A. F. Spivak, Y. A. Dzenis and D. H. Reneker, Mech. Res. Commun., 2000, $27,37$.

8(a). C. Shao, H. Kim, J. Gong and D. Lee, Nanotechnology, 2002, 13, 635; (b) H. Dai, J. Gong, H. Kim and D. Lee, Nanotechnology, 2002, 13, 674.

9(a) .G. Larsen, R. Velarde-Ortiz, K. Minchow, A. Barrero and I.G. Loscertales, J. Am. Chem. Soc., 2003, 125, 1154;

(b).I. G. Loscertales, A. Barrero, M. Marquez, R. Spretz, R. Velarde-Ortiz and G. Larsen, J. Am. Chem. Soc., 2004, 126, 5376; (c)D.Li and Y. Xia, Nano Lett., 2004, 4, 933; (d) J. Watthanaarun, V. Pavarajarn and P. Supaphol, Sci. Technol. Adv. Mater., $2005,6,240$.

10 (a) C. Sanchez, B. Lebeau, F. Ribot and M. In, J. Sol-Gel Sci. Technol., 2000, 19, 31;

(b) C. J. Brinker, R. Sehgal, N. K. Raman and S. S. Prakash, Mater. Res. Soc. Symp. Proc., 1995, 368, 329.

11.See for example, effort by Donaldson company , www.donaldson.com/en/filtermedia/support/datalibrary/003972.pdf.

12. G. Larsen, National Institutes of Health-NIEHS Award No. 1 R43 ES013367-01 A1. 13. D. Li and Y. Xia, Nano Lett., 2003, 3, 555.

14. M. Yoshida and P. N. Prasad, Chem. Mater., 1996, 8, 235.

15 .K. S. Finnie, V. Luca, P. D. Moran, J. R. Bartlett and J. L. Woolfrey, J. Mater. Chem., 2000, 10, 409.

16 . F. X. Perrin, V. Nguyen and J. L. Vernet, J. Sol-Gel Sci. Technol., 2003, 28, 205.

17 (a) .D. P. Birnie, III and N. J. Bendzko, Mater. Chem. Phys., 1999, 59, 26; 
(b) D. P. Birnie, III, J. Mater. Sci., 2000, 35, 367.

18 S. Doeuff, M. Henry, C. Sanchez and J. Livage, J. Non-Cryst. Solids, 1987, 8, 206.

19 A. Bozkurt and W. H. Meyer, J. Polym. Sci., Part B: Polym. Phys., 2001, 39, 1987.

20 (a) S. Barboux-Doeuff and C. Sanchez, Mater. Res. Bull., 1994, 29,1; (b) U. Schubert, J. Mater. Chem., 2005, 15, 3701.

21 P. D. Moran, G. A. Bowmaker, R. P. Cooney, K. S. Finnie, J. R. Bartlett and J. L. Woolfrey, Inorg. Chem., 1998, 37, 2741.

22 E. L. Patrick, C. J. Ray, G. D. Meyer, T. P. Ortiz, J. A. Marshall,J. A. Brozik, M. A. Summers and J. W. Kenney, III, J. Am. Chem. Soc., 2003, 125, 5461.

23 J. J. Feng, Phys. Fluids, 2002, 14, 3912.

24 M. M. Hohman, M. Shin, G. Rutledge and M. P. Brenner, Phys.Fluids, 2001, 13, 2221.

25 (a) P. Viswanathamurthi, N. Bhattarai, C. K. Kim, H. Y. Kim and D. R. Lee, Inorg. Chem. Commun., 2004, 7, 679; (b) J. Yuh, J. C. Nino and W. M. Sigmund, Mater. Lett., 2005, 59, 3645; (c) B. Ding, Ch. K. Kim, H. Y. Kim, M. K. Seo and S. J. Park, Fibres Polym., 2004, 5, 109; (d) B. Ding, H. Kim, Ch. Kim, M. Khil and S.Park, Nanotechnology, 2003, 14, 532; (e) G. Zhang, W. Kataphinan, R. Teye-Mensah, P. Katta, L. Khatri, E. A. Evans, G. G. Chase, R. D. Ramsier and D. H. Reneker, Mater. Sci. Eng., B, 2005, 116, 353. 